Review

\title{
Overview of Space Debris Mitigation Activities in ISRO
}

\author{
Santosh Kosambe \\ Independent Researcher, Pune, India
}

Article history

Received: 29-06-2019

Revised: 02-07-2019

Accepted: 12-07-2019

Email: santo2040@gmail.com

\begin{abstract}
For several decades orbital debris has been identified as a serious concern by all space-faring agencies and nations as it potentially threatens the current and future space endeavors. The Indian Space Research Organization (ISRO) is well aware of the present space debris scenario and is aimed towards achieving the goal of preserving outer-space for humanity. ISRO works on different aspects to effectively manage the threats due to space debris. This paper provides an overview of past activities carried out in ISRO towards the implementation of space debris mitigation guidelines. One of the significant step taken is the implementation of end of life passivation of the cryogenic upper stage of ISRO's Geosynchronous Satellite Launch Vehicle (GSLV). Another one is the successful design and development of propellant venting system for the upper stage of ISRO's Polar Satellite Launch Vehicle (PSLV). ISRO's constellation of communication and navigation satellites orbiting in GSO's are designed with adequate propellant so that it can re-orbit to the higher graveyard orbit at the end of their operational lifetime. A typical successful re-orbiting and decommissioning operation of INSAT-3E is described in detail. ISRO has successfully designed and developed the models and software to predict the atmospheric re-entry of satellites and launch vehicle upper stages, and also to compute the collision risk between the debris and the operational satellites. ISRO has also developed models to study the evolution of the space debris environment in LEO and GEO region. ISRO enthusiastically works with the United Nations Committee on the Peaceful Uses of Outer Space (UNCOPUOS) and Inter-Agency Space Debris Coordination Committee (IADC) to further improve the space debris mitigation guidelines.
\end{abstract}

Keywords: ISRO, Space Debris, Mitigation, Debris Modeling, Re-orbiting, Passivation, Collision Avoidance

\section{Introduction}

Established in 1969, the ISRO has the vision to develop and harness space technology in national development, while pursuing planetary exploration and space science research. Since then, ISRO has gained a place among the elite space agencies in the world over the years, by successfully demonstrating its unique and cost-effective technologies. The prime responsibility of ISRO is design, development, and realization in the advancement of space science technologies and applications towards achieving self-reliance and facilitating in the all-round development of the nation. With these objectives, ISRO has established five significant constellations of space systems, such as Indian National Satellite (INSAT) program, GSAT program, Indian Remote Sensing (IRS) program, GPS Aided Geo Augmented Navigation (GAGAN) program, and Indian Regional Navigation Satellite System
(IRNSS) program. The INSAT program is developed to provide services such as telecommunication, meteorological, television broadcasting, developmental education, societal applications such as telemedicine, tele-education, tele-advisories, and so on. Out of twenty four satellites launched, eleven satellites are still operating in their respective orbits. The GSAT program is developed for digital audio, video, and data broadcasting. Total of nineteen satellites were launched out of which sixteen satellites are still operational. The IRS satellite program is developed for the management of natural resources, Earth observations and various developmental projects across the country. Currently, thirteen operational satellites are orbiting in Sun Synchronous Orbit (SSO) and four in Geostationary Orbit. Also, the launch vehicles for the program mentioned above i.e., PSLV and GSLV were developed to place these constellations of satellites into desired orbits around the Earth. The PSLV and GSLV have successfully launched several Earth observation and 
communication satellites not just for India but for other countries as well.

Mars Orbiter Mission (Mangalyaan-1 aka MOM), the genuinely maiden first interplanetary spacecraft mission of ISRO, launched on November 5, 2013, successfully got inserted into Martian orbit on September 24, 2014, in its first attempt. MOM has successfully completed 1,810 days in its orbit around Mars, though the designed mission life of MOM was six months. MOM still continues to provide vital information about Mars regularly. Most recent and remarkable space probes of ISRO include Chandrayaan-1 and AstroSat. ISRO's lunar orbiter mission (Chandrayaan-1) performed highresolution remote sensing of the moon in visible, nearinfrared (NIR), low energy X-rays and high-energy Xray regions. India's first dedicated Indian Astronomy Satellite (AstroSat) aimed at studying celestial sources in X-ray, optical and UV spectral bands simultaneously. Also, the upcoming missions of ISRO are Chandrayaan2 , the second mission to the moon comprising of an orbiter, lander, and rover to conduct the new experiments on moon's surface was launched on July 22, 2019, and Aditya-L1 will be launched in 2020 to study the process of coronal heating and other phenomena in the magnetosphere region of the sun (ISRO, 2018).

Woefully, throughout this space era, a significant amount of space debris has left behind by India as well as space initiative nations. These objects no longer serve any useful functions, but potentially threatens future space endeavors planned or yet to be planned. Thus, space debris becomes a critical issue to deal with among all space-faring nations. This paper provides an overview of earlier activities carried out in design and development towards the implementation of space debris mitigation guidelines.

\section{Earlier Space Debris Mitigation Activities in ISRO}

The space debris mitigation guidelines in ISRO have been implemented in mission planning, design, manufacture, and operational phases of the satellite program and launch vehicle orbital stages. The constellation of satellites like IRNSS, GAGAN, GSAT, IRS, INSAT, and the satellite launch vehicles PSLV and GSLV, as well as other space systems, were designed in such a way that no debris will be created during the operational phases. Also, appropriate precautions are taken in designing such space systems to avoid failure modes which may lead to accidental break-ups resulting in an increment of debris. Further, development in the designed tools and models helps ISRO to predict the probability of an accidental collision with the operational satellites or orbital debris during the launch and operational phases of the space systems. The analysis of such instances is carried out regularly at satellite control centers. The models are helpful during the relocation of operational as well as non-operational satellites into different orbits. The tool also plays a vital role in planning the launch window as well. Notably, the adjustment in the launch time of PSLV32 in March 2016, PSLV-C23 in June 2014, and PSLV-C4 in September 2002 was done to avoid the possibility of a potential collision with the orbital debris.

In order to minimize the risk of on-orbit fragmentations, ISRO successfully employs a propellant venting system in the final stage of PSLV and passivation of the Cryogenic Upper Stage (CUS) of GSLV. The launch vehicles are designed with the propulsion system as integrated systems. Also, the fuel used is liquid propulsion, and ejecta does not contain any solid residual. ISRO's constellations of satellites in and near GSO and GEO region, are designed with adequate propellant margins to reduce the potential for future collisions by re-orbiting them to a higher graveyard orbit at the end of their operational lifetime. All forms of stored energy are safed in order to prevent an in-orbit explosion (Adimurthy and Ganeshan, 2006). A number of analytical models of fragmentation events have been designed and developed to compute the evolution of breakups in orbits (Ganeshan et al., 1988; Ganeshan and Ananthasayanam, 1996; 1997; Ganeshan et al., 2001; Ananthasayanam et al., 2003; Anil Kumar et al., 2002; 2003; Ananthasayanam et al., 2002; Sharma et al., 2002; Anil Kumar and Subba Rao, 2002; Bandyopadhyay et al., 2004). A detailed study of hypervelocity impacts on space systems is done using finite element techniques (Shone, 2003). All the space debris mitigation activities followed in ISRO are as per the ISO norms 24113:2019 (ISO, 2019). Also, the design and operations of all the satellites and launch vehicles are strictly followed as per the IADC and UNCOPUOS guidelines (IADC, 2007) (UNOOSA, 2010). ISRO actively participates in the various activities of UNCOPUOS and IADC related to the space debris. Also, as an active member of the committee ISRO hosted the 21st and 28th IADC Meeting during March 2003 and March 2010 respectively.

\section{Upper Stage Passivation}

Explosions of satellites, spacecraft and launch vehicle upper stages in orbits around Earth creates a tremendous amount of space debris. By far, $74.8 \%$ of the cataloged space debris population has originated from such events. About 242 orbital fragmentations have been reported so far, with $44.2 \%$ of these breakups are known to have propulsion-related causes. Approximately $3.7 \%$ were due to batteries, $2.5 \%$ were caused by an aerodynamic breakup, and $24.4 \%$ were the result of deliberate actions that are thought to be related to national security (NASA, 2019). A pictorial representation of fragmentations of satellites and rocket bodies in orbits around the Earth is shown in Fig. 1. 


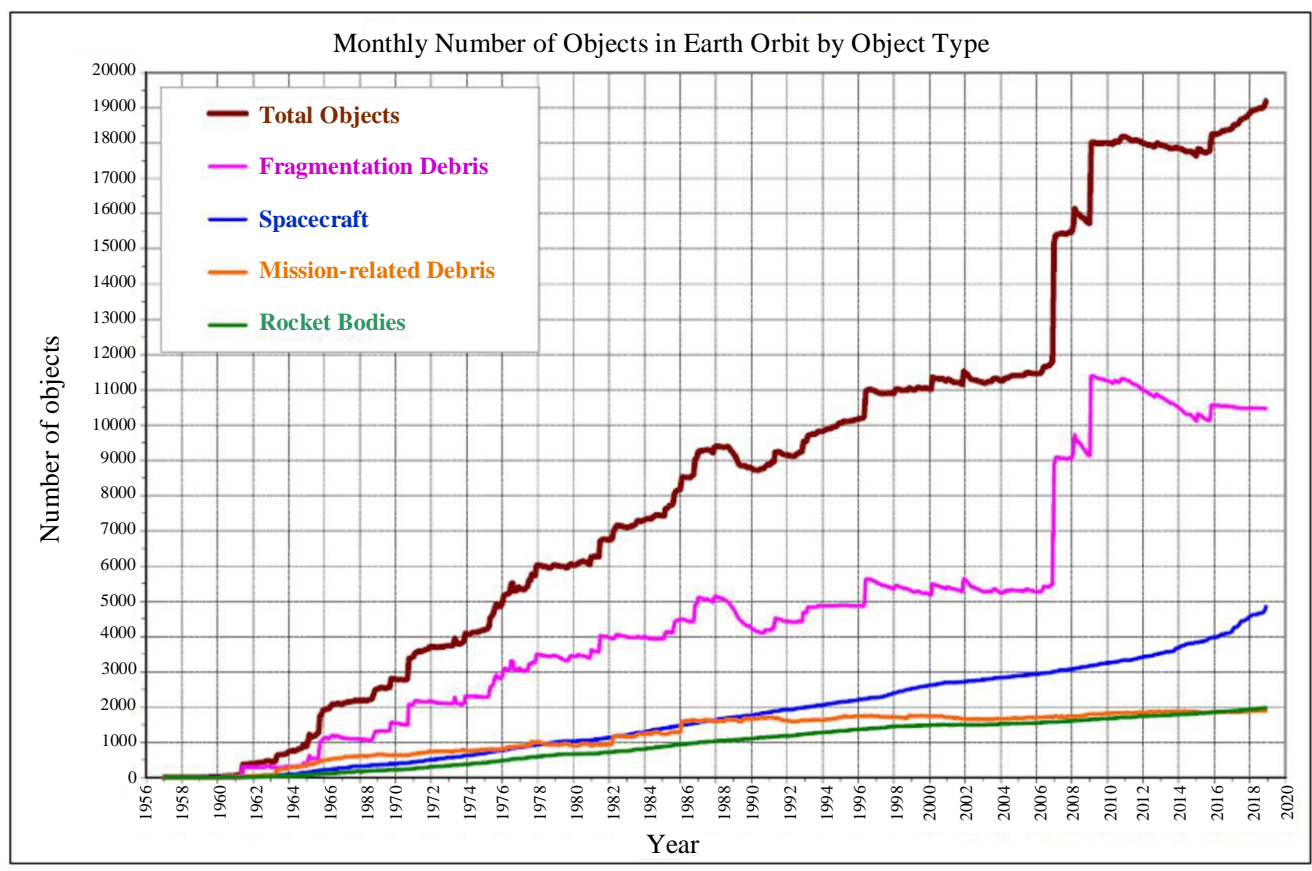

Fig. 1: Monthly Number of Objects in Earth Orbit Cataloged by the U.S. Space Surveillance Network.

Table 1: Top worst satellite breakups in the past cataloged by U.S. space surveillance network

\begin{tabular}{lcll}
\hline Object name & Cataloged Debris & Year of Event & Reason for Breakup \\
\hline Microsat-R & 400 & 2019 & Intentional \\
Iridium 33 & 567 & 2009 & Collision \\
Cosmos 2251 & 1559 & 2009 & Collision \\
Cosmos 2421 & 509 & 2008 & Unknown \\
Fengyun-1C & 3216 & 2007 & Collision \\
PSLV-C3 Rocket Body & 330 & 2001 & Explosion \\
TES Rocket Body & 370 & 2001 & Explosion \\
CBERS 1 Rocket Body & 343 & 2000 & Explosion \\
STEP 2 Rocket Body & 710 & 1996 & Explosion \\
SPOT 1 Rocket Body & 492 & 1986 & Explosion \\
Nimbus 4 Rocket Body & 375 & 1970 & Explosion \\
OV-2-1/LCS-2 Rocket Body & 473 & 1965 & Explosion \\
\hline
\end{tabular}

The majority of these breakups were unintentional, many arise from a wide variety of reasons, including propellant tank explosions due to the mixing of fuel and oxidizer, battery rupturing, thruster malfunctions, tank failures due to impact of small debris, degradations of the structure, accidental collisions, over pressurization of fuel tanks, deliberate explosions and many more. Some major breakup events observed in the past were recorded in Table 1.

Detailed study and analysis of past incidents shows that the most effective method to stop such events is by removing of all forms of stored energy from spacecraft and launch vehicle upper stages at the end of their mission, also known as passivation. In order to limit the risk of on-orbit fragmentations, all onboard sources of stored energy should be depleted or safed when no longer required. The process includes removal of residual propellants by either venting or burning, the expulsion of compressed fluids, discharge of electrical storage devices, disabling of momentum wheels and communication devices, and safing of any destruct devices, etc. Though the risk associated with the on-orbit explosion were known, the incident of PSLV-C3 occurred. After the explosion of PSLV-C3, successful implementation of passivation of the launch vehicle upper stage is carried out in the design of PSLV from C4 mission onwards (Adimurthy and Ganeshan, 2006).

The potential solutions envisioned for the passivation of the 4th stage of PSLV is presented during the 21st IADC in 2003, which includes:

(a) Venting the trapped propellants and subsequently the pressurant through the main engine in a sequential manner by opening the main engine valves.

(b) Consuming the total propellants by restarting the main engines. 
(c) Consuming the propellants by firing the reaction control thrusters meant for attitude stabilization.

(d) Venting the propellants through an additional branching in the feed lines of each propellant using separate pyro valves added in the circuit.

(e) Venting the pressurant gas from the propellant tank and gas bottles along with the propellant vapors in the tanks through an additional branching in the pressurization lines of each tank using separate pyro valves added in the circuit.

By considering the safety measures and simplicity of operation, the last proposed option was considered for the passivation of PSLV-C4. Passivation begins with disabling recovery mechanisms, followed by passivation of the propulsion, attitude control, power and communication systems. As each system is disabled, its contribution to the mission is nullified. While bearing in mind a proper order of the steps is designed while planning the passivation procedure.

Further, Adimurthy and Ganeshan (2006) explains the problem areas addressed, and required actions taken in the implementation of passivation schemes in spacecraft and launch vehicle upper stages at ISRO:

1. For avoiding buckling of the common bulkhead in the propellant tanks during passivation, positive pressure was maintained in the MMH tank by venting the MON-3 tank first.

2. To successfully implement the last suggested option, two separate pyrotechnical valves have been introduced in the pressurization circuit of $\mathrm{MMH}$ and MON-3 tanks.

3. To avoid interaction of the exhaust plume with the structure, vent nozzles was positioned 180 apart to eliminate the interaction zone between them.

4. To eliminate the reaction torques during the process of passivation and achieve the reaction less system, two nozzles were kept in the opposite direction to one another.

5. Contamination of the spacecraft was avoided by providing sufficient time for the separation of spacecraft before initiating the process of passivation.

6. Experiments were carried out with MON-3 gas in high altitude test facility to ensure no freezing of propellants during the passivation.

7. To avoid explosions related to batteries, the solar arrays were utterly disconnected from the main power bus by switching off the charging lines at the end of the mission, and the remaining energy is utilized for performing the passivation operations.

8. To avoid any interference with the neighbor satellites, the communication systems were switched off completely.

9. In the case of PSLV-C4, it is impossible to achieve complete passivation due to the presence of common bulkhead in the propellant tanks. So, it is recommended to lower its pressure significantly below its critical pressure i.e., the pressure below which the stress in the tank wall is such that a crack does not propagate violently, but remains confined; a hole would therefore not degenerate into an explosion. It can be considered that the critical pressure of a tank is of the order of half its burst pressure. Also, besides theoretical analysis and calculation, all kinds of pressure vessels simulations and hypervelocity impact set-ups had done to ensure that no break-ups would occur at this critical pressure. Pressure measurements from MON and MMH tanks recorded in Fig. 2 shows the successful passivation of the PSLV-C4. After the fragmentation of the PSLV-C3 and implementation of passivation, none of the ISRO's satellite or launch vehicle upper stage exploded in space creating any debris due to on-orbit explosions and collisions.
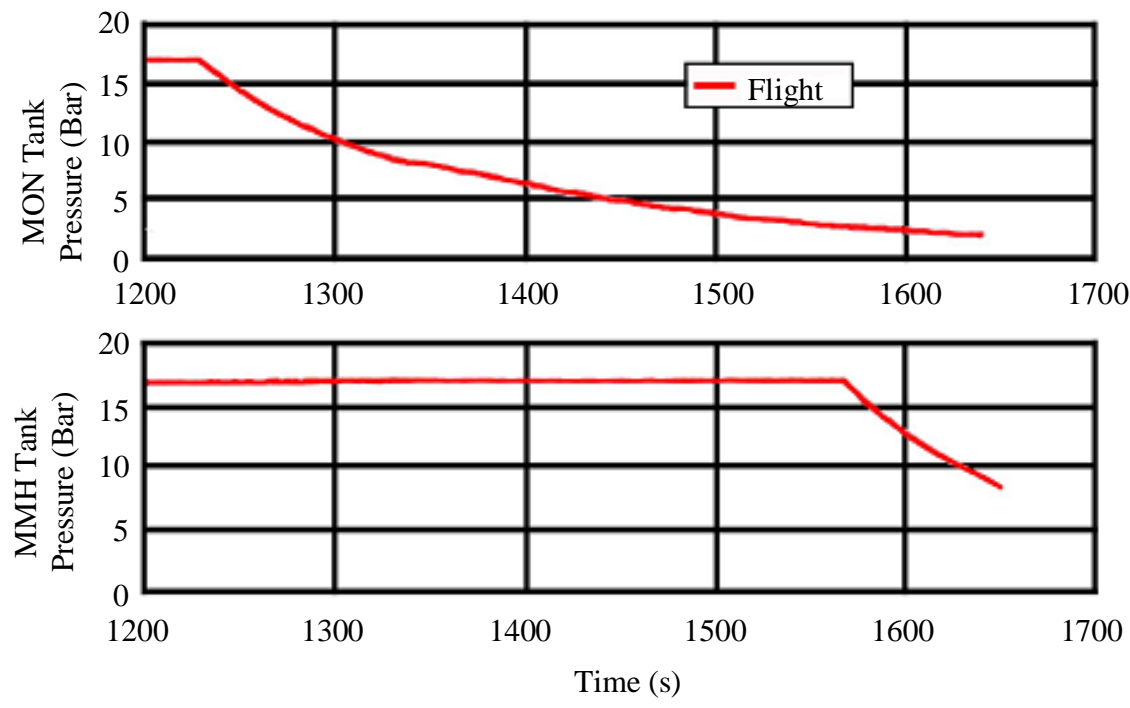

Fig. 2: Pressure variation in MON and MMH tank recorded by PSLV during passivation 


\section{Space Object Re-Entry Estimation}

Thousands of satellites, rocket bodies and fragments are currently orbiting around the Earth. All these objects tend to reenter the Earth's atmosphere because of the atmospheric drag. On average, two small tracked objects reenter the Earth's atmosphere each day. So far, most of the re-entries happened till date are in an uncontrolled fashion. Fortunately, there have been no known reports of injuries to people or damage to the property from reentering space debris. However, in general, reentering objects pose a high risk to people and infrastructure on the ground and aviation. For safety, it is essential to estimate the impact of such uncontrolled re-entries. Also, re-entry of space objects or spacecraft is the most preferred method for space debris mitigation in LEO. It is challenging to predict that debris from a randomly reentering satellite will hit Earth because of the uncertainties in the orbital propagation model, atmospheric density models, solar flux, geomagnetic activity indices, and further observation of orbital parameters. In general, we can predict the time that re-entry will begin with the uncertainty of $10 \%$ of the actual time. Unfortunately, re-entering objects travel so fast that a minute of error in the time is equivalent to hundreds of miles on the ground. Hence, the accurate estimation of the predicted re-entry time and impact location of decaying space debris or objects is necessary for proper planning of mitigation strategies and hazard assessment. Due to the criticality involved in the re-entry of such objects, ISRO has developed several suitable mathematical models to evaluate the trajectory and destruction process of spacecraft and rocket bodies during re-entry. The various tools have been developed to predict the final orbital characteristics e.g., re-entry time, impact location, target velocity, etc. for LEO based satellites as well as launch vehicle upper stages GSLV and PSLV re-entering the Earth's atmosphere.

The ISRO utilizes a mathematical model developed for the online re-entry prediction of its satellites and launch vehicle orbital stages which use constant gain kalman filter approach to estimate the ballistic parameter. A simple model is developed for the orbit propagation with the filter states being the semi-major axis, eccentricity and ballistic coefficient. The measurements of the apogee and perigee are derived from the Two Line Elements (TLE) data provided by agencies like USSPACECOM. The primary feature of the model is that any un-modeled and un-modellable state and measurement errors can be accounted by adjusting the kalman gains which are selected based on a suitable cost function. In this model, the mean US standard atmosphere and a simple propagator considering only the atmospheric drag effects were used.

Also, the model estimates only the semi-major axis and eccentricity decay in one revolution by considering only constant scale height during the revolution. The accuracy in the re-entry prediction can be increased by using the more sophisticated orbit propagators with high fidelity atmospheric models (Anil Kumar et al., 2007). The validation of this method is done by utilizing three re-entries of debris objects, namely SROSS-C2 Satellite, COSMOS 1043 and Soyuz 11A511 U rocket body. The re-entry prediction obtained from the proposed model is nearly close to the actual re-entry dates. Unfortunately, the predicted results depend upon the latest TLE data set available from USSPACECOM and the specific modeling of the ballistic coefficient. One of the drawbacks of the model is that it only utilizes a mean atmosphere and a simple propagator which only consider atmospheric drag effects. The model has to be redesigned such that it can handle the latest atmospheric models as well as complex propagators. The many perturbations also have to be included in the model to predict the exact location, time, and date for the reentering objects. Sharma and Anilkumar (2005) further tried to improve the above model by incorporating a more accurate propagation model based on Kustaanheimo-Stiefel element equations. The constant kalman gains were estimated by minimizing an objective function through a genetic algorithm.

An integrated model consisting of a high-precision orbit propagator (HPOP) and a multi-objective function optimization technique is developed for the prediction of accurate re-entry time of any object during the last phase of orbital decay. The model estimates an Essential Ballistic Parameter (EBP) by minimizing a multiobjective function of errors on the predictions concerning observed values of orbital parameters of the decaying object at various epoch. Least-square errors, weighted least-square errors, considering the expected remaining life as weights, and normalized nondimensioned errors are taken as the components of the multi-objective function. EBP absorbs the uncertainties in TLEs, propagation and atmospheric model inaccuracies, and other model input uncertainties. The orbital parameters are computed from TLEs using Simplified General Perturbations No. 4 (SGP4) theory at their epoch (Shraddha and Anilkumar, 2015). The validation of this proposed model is done with the help of decayed objects Phobos Grunt and ROSAT, and the predicted results show an excellent match with the first re-entry time. The newly designed model can overcome the drawbacks of the previously proposed models by handling the inaccuracies in TLEs during the last phase of orbital decay. Also, the model can handle the various atmospheric models, complex propagators, and induced perturbations as well. Although, the model is only limited to predict the re-entry of objects during the last phase of decay and the accuracy of prediction has direct dependability on the selection of the number of TLEs or period of TLEs to be used. It has to be further improved to predict the re-entry of 
an object throughout their life by modifying the orbit propagators and multi-objective function.

Various models are also developed to predict the reentry time of decaying space objects from LEO such as Response Surface Method (RSM) with genetic algorithm (GA), Optimization with Satellite Tool Kit (STK OPTIM), and Lifetime Optimization using Satellite Tool Kit (STK LTOptim) (Anilkumar et al., 2017). In all these models, estimation of EBP has been carried out, which subsequently results in an optimal re-entry time. HPOP with an optimization technique is also used for the accurate prediction of re-entry time in these models. Also, several Object-oriented as well as Spacecraft oriented codes like Debris Assessment Software (DAS), Object Oriented Surveillance Analysis Tool (ORSAT) developed by NASA, and Spacecraft Atmospheric Reentry and Aerothermal Breakup (SCARAB) developed by ESA are also used to perform the re-entry analysis of Spacecraft and launch vehicle orbital stages.

One of the above model is used to carry out the reentry analysis of PSLV-C39/IRNSS 1H, which was launched on August 31, 2017. The results obtained from the TLE data set predicted that the re-entry would occur in between March 2, 2019, to March 3, 2019, while the object decayed on March 3, 2019. Although, the designed and developed models are quite useful to predict the re-entry of any objects orbiting in space, sometimes ISRO has to take help from the international organizations and space agencies to calculate and predict the re-entries of some objects. Also, ISRO provides information on its upcoming and past re-entries to a broad target audience, including national protection agencies, researchers, and the general public. ISRO participates in and hosts a re-entry data exchange platform for the IADC as well. The developed re-entry models plays an essential role in the successful demonstration of ISRO's two re-entry missions, namely, Space Capsule Recovery Experiment (SRE-1) and Crew module Atmospheric Re-entry Experiment (CARE).

\section{Modeling of Space Debris Environment}

The cataloged debris population does not include the numerous events that are too small for tracking from ground stations. Such objects pose a higher risk to the current as well as future space endeavors. Hence, modeling of the space debris population is essential to describe the traceable as well as untraceable orbita close approach of such objects to the operational satellites, and to predict the re-entry of satellites. Also, it is needed to fill in gaps in the existing cataloged data, to interpret new data, and to project the characteristics of the future debris environment. ISRO designed and developed such mathematical models to carry out in-depth studies in the areas of space debris modeling which includes breakup modeling, environment modeling, and evolution of debris environment, collision probability analysis, and re-entry prediction. Some of the developed models are A Semi-Stochastic Environment Modeling of Breakup in LEO (ASSEMBLE), Stochastic IMPressionistic Low Earth (SIMPLE), Stochastic IMPressionistic GEO Environment (SIMPGE), Spatial density modeling in LEO using wavelets and continuous wavelets transforms, evolution of space debris scenario based on the propagation of the characteristics of equivalent fragments, prediction of re-entry of satellites and objects based on kalman gain filter, and the orbital debris environment in GEO (Ganeshan et al., 2007). 1 debris environment. The mathematical models are used to carry out the analysis of risk due to the space debris, close approach of such objects to the operational satellites, and to predict the re-entry of satellites. Also, it is needed to fill in gaps in the existing cataloged data, to interpret new data, and to project the characteristics of the future debris environment. ISRO designed and developed such mathematical models to carry out in-depth studies in the areas of space debris modeling which includes breakup modeling, environment modeling, and evolution of debris environment, collision probability analysis, and re-entry prediction. Some of the developed models are A Semi-Stochastic Environment Modeling of Breakup in LEO (ASSEMBLE), Stochastic IMPressionistic Low Earth (SIMPLE), Stochastic IMPressionistic GEO Environment (SIMPGE), Spatial density modeling in LEO using wavelets and continuous wavelets transforms, evolution of space debris scenario based on the propagation of the characteristics of equivalent fragments, prediction of re-entry of satellites and objects based on kalman gain filter, and the orbital debris environment in GEO (Ganeshan et al., 2007).

An ASSEMBLE model is designed to simulate the on-orbit breakups of satellites and space objects. The model depends on the data of the breakup events and characterization of orbital parameters such as semi-major axis, eccentricity, inclination, size, shape, and ballistic coefficient cataloged by USSPACECOM. Upon analysis of the TLE data set of some of the fragmentations, it is assumed that at the time of the breakup the orbital parameters obey certain statistical distributions such as the apogee or perigee height, and the inclination follow Laplace distributions, while the eccentricity and the ballistic coefficient follows a Lognormal distribution. The location and scale parameters of such fragments depend on the orbit of the parent body at the time of breakup and intensity of the explosion, respectively. The model utilizes such data to define orbital characteristics of the fragments (Anilkumar et al., 2005). The simulation results obtained from the proposed model are comparable quite well to simulate the on-orbit breakups of objects in LEO.

The debris scenario up to an altitude of $2000 \mathrm{~km}$ and eccentricity from 0 to 0.2 is described in the SIMPLE 
model of the space debris environment. The model is capable of covering about $75 \%$ of the large space debris objects of TLE data sets cataloged in USSPACECOM. The probability distribution function and the statistics of the various characteristics data observed at various times are used to model the randomly evolving dynamical process of space debris environment. The method utilizes two types of models called gross and local, respectively. The gross model provides insight into the physical process of objects for all inclinations over the whole LEO region by characterizing the distribution of the eccentricity, number density, and the ballistic coefficient. While, the local model characterizes the distribution of ballistic coefficient and eccentricity of the objects at suitable altitude and perigee height important for risk assessment and mission analysis. The gross and local model represents the number density, eccentricity, and ballistic coefficient in terms of Laplace distributions and lognormal distributions, respectively. The model is designed with fewer parameters to closely capture all the peak fragment densities with accuracy at other altitudes and is used to estimate the spatial density of objects, collision probability, and flux at each altitude (Ananthasayanam et al., 2006a). The proposed model is best suitable to predict the orbital debris scenario in LEO below the altitude of $2000 \mathrm{~km}$. The only drawback is that the model cannot be utilized for debris scenario in higher orbits and with an eccentricity greater than 0.2.

The evolution of space debris scenario is represented using the propagation of the characteristics of an equivalent fragment without propagating every individual debris fragment, together with a constant gain kalman filter technique. The equivalent fragments in each of the bins are assigned to a three-dimensional bin of semi-major axis, eccentricity, and an equivalent ballistic coefficient (a, e, B). The model can provide the suitable ballistic coefficient values for the equivalent fragments in the various bins. Also, it can assimilate the measurement information over time from other breakups as well. The constant kalman gain approach can track the dynamically evolving fragment scenario and further expand the scenario to provide an appropriate timevarying equivalent ballistic coefficient for the equivalent fragments in the various bins. Also, it helps to minimize the errors due to the finite bin size and the environmental perturbations (Ananthasayanam et al., 2006b). The Spatial density model is designed to perform the conjunction analysis between the operational satellites and space debris objects for possible mitigation, prevention, or maneuver (Anilkumar and Reddy, 2009). An algorithmic procedure using wavelets is used to automatically estimate the exact model parameters corresponding to the peak location and number of peaks. The wavelets speed up the parameter estimation process for the models with peaks (Reddy et al., 2011).

The statistical distribution nature of the orbital characteristics such as semi-major axis, eccentricity and ballistic coefficient of the tracked non-operational GEO debris is obtained with the help of SIMPGE Model. The model is based on the approach and philosophy as laid out for SIMPLE model for LEO (Anilkumar et al., 2005). The constant gain kalman filter approach is designed to predict the re-entry time of space objects which can handle numerous uncertainties, sophisticated features, and variables existing in the space debris environment (Anilkumar et al., 2007). The simple propagator by considering drag alone is used to obtain the constant gain. A long-term numerical propagator is also developed for the ISRO's communication satellites to study perturbation due to Solar Radiation Pressure (SRP), third body effects due to luni-solar forces, Earth's gravitation potential, and perigee height variation for years.

\section{Collision Avoidance Analysis}

In the present scenario, the number of satellites orbiting around the Earth is increasing, even though some of them re-enters into the Earth's atmosphere. As on May 2019, Orbital Debris Quarterly News (NASA, 2019), shows that there are 19,404 objects are present in the earthbound space in which only 4,972 are the operational and defunct satellites. The remaining objects existing are the orbital debris formed due to the end of life, fragmentation, collision, spacecraft anomalies, and things left by astronauts during extra-vehicular activities. In case of GSO region, there are 1,523 objects out of which 519 are controlled in their longitude slot, as on May 2018 reported in (ESOC, 2018). Ever increasing orbital debris has become a significant concern for the space-faring nations, since it possesses a potential threat to the operating satellites. A collision or explosion in space would increase the number of cataloged objects catastrophically; also such an event could damage an operating satellite, resulting in technological as well as economic loss.

Several on-orbit collisions in the past has increased the tremendous amount of space debris population in space. Hence, it is essential to protect satellite and launch vehicle in its ascent and orbital phase, as well as spacecraft upon injection to prevent the further generation of space debris through collision. The SPAce DEbris PROximity (SPADEPRO) tool is developed to perform the collision avoidance or COLA analysis for the powered and the orbital phase of ISRO's satellites as well as launch vehicles. SPADEPRO referred to assessment of collision risk between cataloged space debris and desired satellite or launch vehicle. The prime objective of COLA analysis is to detect close approaches of debris to satellites and launch vehicles during the launch and early post-deployment. If the maximum collision probability exceeds 1 in 1000 or the minimum conjunction distance falls below $100 \mathrm{~m}$ SOPA protocol is implemented, and collision avoidance maneuver is 
performed for satellites in orbit. Also, the required adjustment in the launch time of the satellite or launch vehicle is made to avoid the potential collision during this period. As shown in Fig. 3, a typical result of Collision Avoidance Analysis or COLA carried out before the launch of PSLV-C14/OceanSat-2, which shows the probability of collision during the launch window.

The TLE data set of trajectory and orbital elements of the desired satellite or launch vehicle essential to perform the SOPA is obtained from USSTRATCOM. The database includes space objects greater than 10 to 30 $\mathrm{cm}$ in LEO and $1 \mathrm{~m}$ in GEO in TLE format. The TLE data format is mainly associated with specific propagation models such as SGP4 and SDP4. It is difficult to estimate the accurate TLE data for the orbital phase of satellites and powered phase of launch vehicles due to the uncertainties involved, as it depends on the number and quality of the measurements, on the orbit exploration model, and on how latest the information is. The TLE data required to perform the analysis of orbital phases is obtained by converting the state vector (altitude, velocity, flight path angle, velocity azimuth, geodetic latitude, east longitude, and epoch) parameters into TLE format. The TGENPRO module has been developed to generate the TLE data set of the satellite or launch vehicle from the state vector. For the powered phase of the launch vehicle in ascent, a TLE data set can be generated by doing some necessary modifications in trajectory parameters (velocity and velocity azimuth). The SPADEPRO analysis depends on the computation of collision probability between desired spacecraft or launch vehicle and cataloged space objects, assessment of acceptable collision probability, and choice of appropriate interval for space debris proximity analysis. The computation of collision probability depends on effective collision radius (RS), combined trajectory dispersion $(\sigma)$, and the threshold for minimum conjunction distance (Rmin). The computation process to determine minimum conjunction distance between the desired satellite and cataloged space object within a specified period is expensive due to the trajectory calculations of all the objects present in the catalog. The process is simplified in order to produce quick results and avoid unnecessary computation, using an orbital separation filter, an apogee-perigee filter, a time filter, and an epoch filter. The use of these filters reduces the number of cataloged objects drastically in the process. The combined trajectory dispersions for carrying out the SOPA analysis for a particular satellite is obtained by carrying out Monte Carlo Analysis.

\section{PSLV-C14/OceanSat-2 Mission COLlision Avoidance (COLA) Analysis} Launch Date : $23^{\text {rd }}$ September 2009

Time Segments Not Cleared for Lift Off during the launch Windows 11:51 IST to 12:06 IST

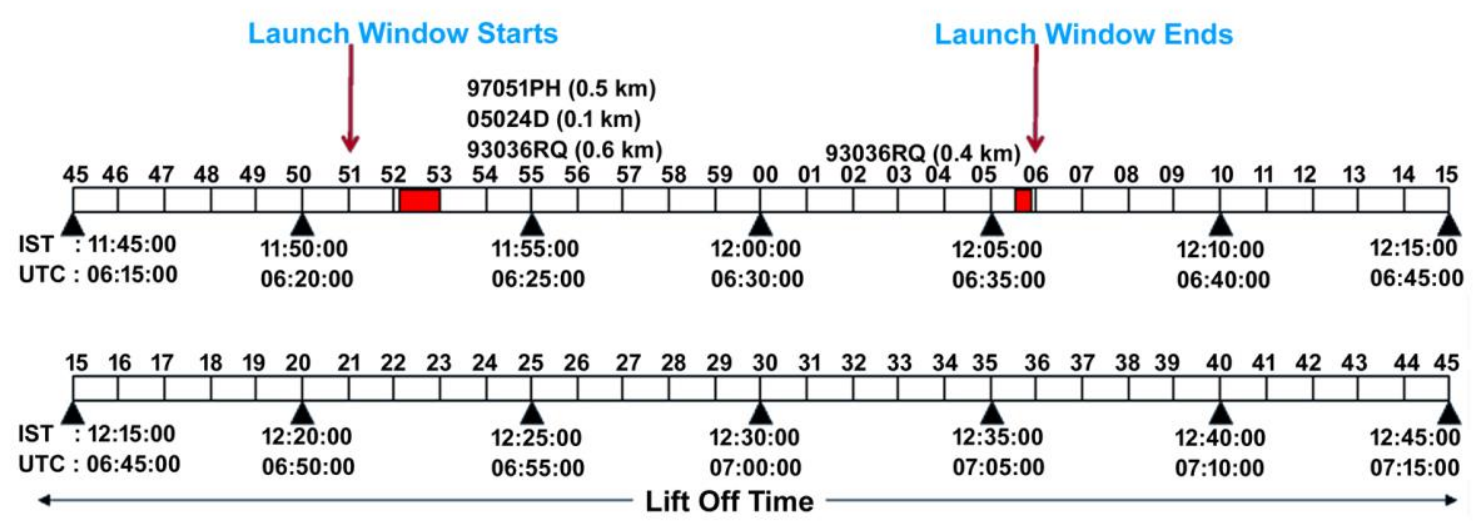

Lift Off not Recommended in Time Intervals Marked

Maximum (Worst Case) Collision Probability Estimated to be More than 1 in 1,000,000 for PSLV-C14 (in ascent Phase) and 1 in 100,000 (Till the completion of first orbit after Injection) for OceanSat-2

Fig. 3: Result of Collision Avoidance or COLA Analysis for PSLV-C14/OceanSat-2 


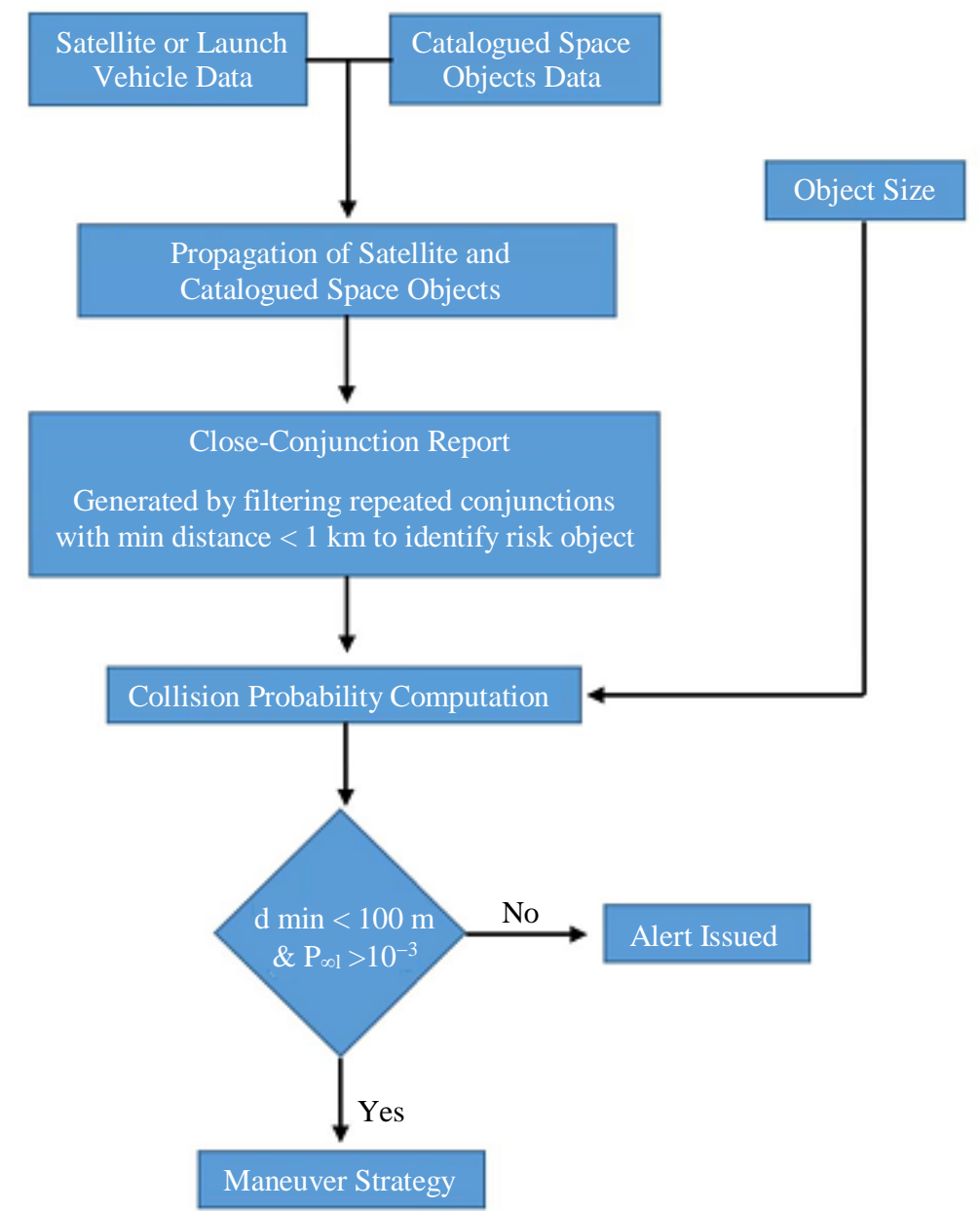

Fig. 4: Typical flow chart of Space Object Proximity Awareness (SOPA)

The trajectory dispersion values of a particular cataloged object can be fixed by considering the type of orbit it presents and the period of the orbital information of the object. The SOPA is carried out at the injection time of satellite for the entire launch windows of about $30 \mathrm{~min}$ duration at $2 \mathrm{~s}$ intervals so that no close approach of the launch vehicle or the satellite with any of the space objects is missed (Bandyopadhyay et al., 2004). The flow chart for typical Space Debris Proximity Analysis is shown in Fig. 4.

As most of the ISRO's navigational, communicational, and metrological satellites are in GEO and GSO orbits, COLA for these satellites are performed regularly. As on January 2019, ISRO is operating twenty seven satellites in GSO for communication, meteorology, and navigation purposes. Among them, nineteen satellites are maintained in geostationary orbit, collocated at four orbital slots. Master Control Facility (MCF) part of ISRO is responsible for monitoring the close approach of objects to these satellites regularly. For the satellites orbiting in GEO and GSO, collision avoidance analysis or COLA is carried out every week based on TLE data obtained from USSTRATCOM. COLA program provides crucial parameters such as time of close approach, minimum distance, and TLE age. If any close approach less than $10 \mathrm{~km}$ is encountered, long term orbital evolution of the close approaching object is studied using historical TLE data, followed by consistency check of the latest TLE update by propagating few of the previous TLEs. The analysis steps are repeated with the subsequent TLE updates until the day of close approach. If the probability of collision is still predicted, the collision avoidance maneuver will be planned for the operational satellite based on minimal delta-velocity at an optimal time, require to achieve a safe distance. Also, the impact on the existing colocation due to collision avoidance maneuver, on-orbit maintenance, and control will be analyzed. In 2015, the five satellites, INSAT-3C, INSAT-4CR, GSAT-14, GSAT-7, and KAPLANA-1 were controlled at 74 East longitude. As per the COLA analysis carried out on July 23, 2005, shown that COSMOS-2440 was approaching 740 East longitude and minimum distance less than 10 $\mathrm{km}$ was predicted. As per the operational guidelines, the 
orbital evolution of COSMOS-2440 was carried out using historical and latest TLE data sets. The detailed analysis carried out using the latest TLE update predicted the relative distance of $7.5 \mathrm{~km}$ with GSAT14 and $4.2 \mathrm{~km}$ with INSAT-4CR. For the other satellites, the relative distance with COSMOS-2440 was found to be higher than $15 \mathrm{~km}$. As a result, along track maneuver with low thrust pulses was carried out to increase the minimum close approach distance. For GSAT-14, the tangential velocity of $0.016 \mathrm{~m} / \mathrm{s}$ and INSAT-4CR, the tangential velocity of $0.027 \mathrm{~m} / \mathrm{s}$ was imparted (Kannan et al., 2015).

\section{End of Mission Re-Orbiting}

ISRO has been operating several constellations of communicational, navigational, and meteorological satellites in GSO and GEO orbital regime. These satellites are designed with adequate propellants for reorbiting to a higher graveyard orbit at the end of their operational lifetime. The decommissioning and reorbiting operation of the communication satellite INSAT-3E is described in detail here. The prime center for spacecraft operations Master Control Facility (MCF) at Hassan is responsible for planning and execution of operations such as orbit determination, orbit raising, orbit maintenance, orbit control, and colocation of GSO and GEO based satellites. INSAT-3E was launched on September 28, 2003, into a GTO with a perigee of 649 $\mathrm{km}$ and an apogee of $36,000 \mathrm{~km}$ inclined at an angle of 70 to the equator. The satellite was successfully repositioned to 54.97 E longitude on October 1, 2003, for continuing the operations. The Liquid Apogee Motor (LAM) was fired in three phases for a total duration of $121 \mathrm{~min}$ to achieve final circular geostationary orbit of $36,000 \mathrm{~km}$.

Unfortunately, on March 29, 2014, INSAT-3E ran out of the on-board oxidizer and stopped working altogether. A decision was taken to decommission the satellite well before its desired lifetime of 15 years. As per the IADC guidelines, the spacecraft that have terminated their mission should be maneuvered to the higher graveyard orbit to prevent interference and onorbit collision with existing satellites or objects in orbit. The required minimum increase in altitude above GSO recommended by IADC is given by:

\section{Altitude $(\mathrm{Km})=235+1000 \times$ Solar Radiation Pressure Coefficient $\mathrm{x}$ Cross-sectional Area/Mass of the satellite}

Before the start of the re-orbiting operation, the satellite orbit was lowered on April 4, 2014, to perigee $35,579 \mathrm{~km}$ and apogee $35,693 \mathrm{~km}$, with eastward drift rate of 2 per day.
The operational procedures followed to re-orbit and decommission the satellite were as follow:

1. At the start of drifting operation computation of close approach to any nearby satellite or COLA was performed while considering the new orbital elements of satellites.

2. The pulsing operation was performed in a controlled way while crossing nearby spacecraft or satellite to avoid the risk of any on-orbit collision or explosion.

3. All the communication devices were switched off completely to avoid any interference to the nearby satellites while drifting.

4. The Nickel-Hydrogen $(\mathrm{NiH} 2)$ batteries and solar arrays were disconnected from the main bus. Further, self-discharge was activated to deplete the amount of stored energy quickly.

5. The balancing devices such as momentum and reaction wheels, magnetic torquers, and thermal loads were turned off. They were kept on earlier for balancing purposes.

6. As a part of passivation, the propulsion valves were closed completely, and it was also ensured that the propulsion tanks are empty.

7. In the end, the Telemetry transponders were switched off to avoid any radio frequency transmission from the satellite.

Though the satellite ran out of onboard oxidizer, the small monopropellant thrusters were used to perform the micro pulsing maneuvers to re-orbit and decommission the satellite successfully. Also, the decommissioning operation of ISRO's communicational satellites such as INSAT-2A, INSAT-2B, INSAT-2C, INSAT-2DT, INSAT-2E, INSAT-3B, GSAT-2, and GSAT-3 was performed as per the IADC guidelines.

\section{Minimization of GTO Lifetime}

A significant amount of space debris resides in the GTO, which is a highly eccentric orbit with the perigee in LEO at an altitude of 180 to $750 \mathrm{~km}$ and the apogee near GSO at an altitude of about $36,000 \mathrm{~km}$. Debris such as launch vehicle upper stages, auxiliary fuel tanks, and payload carriers left in GTO poses a risk to the operational satellites as it frequently traverses LEO and GSO. With the successful launch of each satellite in GEO one or more large pieces of debris is generated and is left in GTO. Hence, minimizing the orbital lifetime of these objects is essential to avoid the risk of on-orbit collisions, and to maintain the clean space environment. These orbits are characterized by periodic changes in perigee altitude caused by the gravitational perturbations due to the Sun and the Moon. The initial orientation of the orbit with respect to Sun and the Moon determines 
the subsequent histories of the orbital evolution. Gravitational perturbations due to the Sun and the Moon directly affect the Right Angle of Ascending Node (RAAN), argument of perigee, and eccentricity of an orbit. The three body perturbations, atmospheric drag, lunar and solar perturbation, and Earth's oblateness influences the orbital evolution of objects in GTO. The combined influence of all these perturbations can result in lifetime variations from a few days to several years. Unfortunately, one cannot always use this natural phenomenon to limit the orbital lifetime of the objects in these orbits. As the launch time of a GSO satellite also depends upon many other factors such as thermal aspects and eclipse time related to the spacecraft design. One of the proposed criteria to ensure a stable space debris environment is to place the objects in orbits with a limited lifetime of up to 25 years. However, the lifetime of orbital objects in GTO can be significantly reduced through appropriate choice of perigee altitude, the longitude of the ascending node, and the launch time of the spacecraft. The lifetime of objects in GTO is affected by a combination of atmospheric drag and luni-solar perturbations or by a luni-solar gravity alone. The atmospheric drag generates a retarding force on the satellite due to which the apogee and perigee of satellite reduces drastically. On the other hand, the perigee altitude associated with the satellite can increase or decrease due to the effect of Sun and Moon. The perigee of the satellite is driven below the decay altitude virtually by luni-solar gravity effect. Due to which complete circularization of the orbit does not take place. As a result, rapid decrease in perigee of the satellite take place and satellite re-enters into the lower atmosphere with a steep entry angle, causing a structural breakup. For highly eccentric orbits like a GTO, the lifetime strongly depends on two parameters, namely, the initial RAAN of the spacecraft and the solar longitude, both of which define Sun azimuth angle on spacecraft orbital plane. These two parameters are functions of time. In the case of lower drag, the decay occurs owing to a sharper decrease in perigee altitude due to the influence of luni-solar gravity. In cases with higher drag, the perigee remains at a relatively higher level as compared to the cases with lower drag. The orbital life of an object in GTO has a strong dependence on the time and date of insertion into the orbit. Even minute changes in the launch time can result in changes of several orders in the lifetime. The interplay of atmospheric drag and luni-solar gravity can lead to a situation where higher drag means more orbital life (Sharma et al., 2004).

The estimation of the orbital lifetime of the cryogenic upper stage of ISRO's GSLV-D5 is discussed. The launch took place on January 5, 2014, which inserted the GSAT-14 into a GTO with mean perigee altitude of $170 \mathrm{~km}$ and apogee altitude of $35,975 \mathrm{~km}$. The state vectors consisting of position and velocity is obtained with the help of SDP4 orbit propagator model by converting the TLE data set of the orbital elements from February 11, 2014, to April 4, 2014. The lunarsolar gravity perturbations, atmospheric drag, solar radiation pressure effects, and Earth's oblateness were considered to obtain the accurate state vectors. The mean and osculating orbital elements at the initial state of the orbit are obtained by providing the state vector as an input to the Numerical Prediction of Orbital Events (NPOE) software. A Response Surface Methodology (RSM) using a genetic algorithm for four intervals of near-linear variation of the mean apogee altitude observed were used to obtain the initial values of the ballistic coefficient, uncertain parameters, and eccentricity. The observed mean apogee and perigee altitudes of GSLVD5 computed from TLE's are plotted in Fig. 5 and Fig. 6. The Earth's gravitational potential up to J10,10 luni-solar point mass gravitation with the Sun and the Moon positions computed from GEM10B.

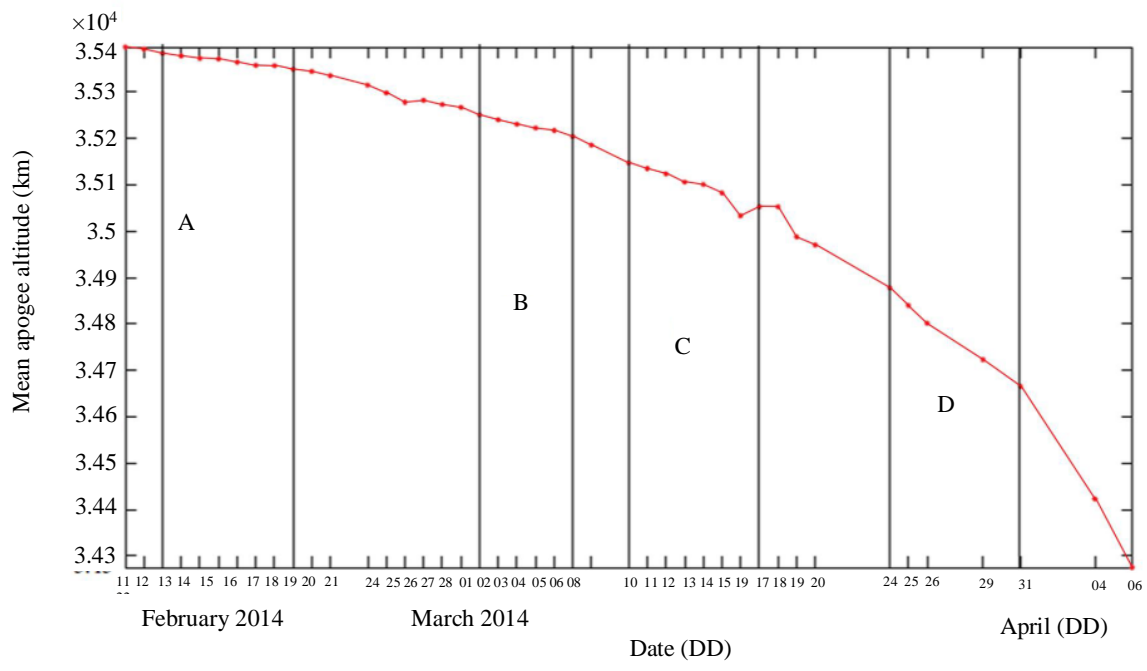

Fig. 5: Variation in computed mean apogee altitude of GSLV Rocket Body 


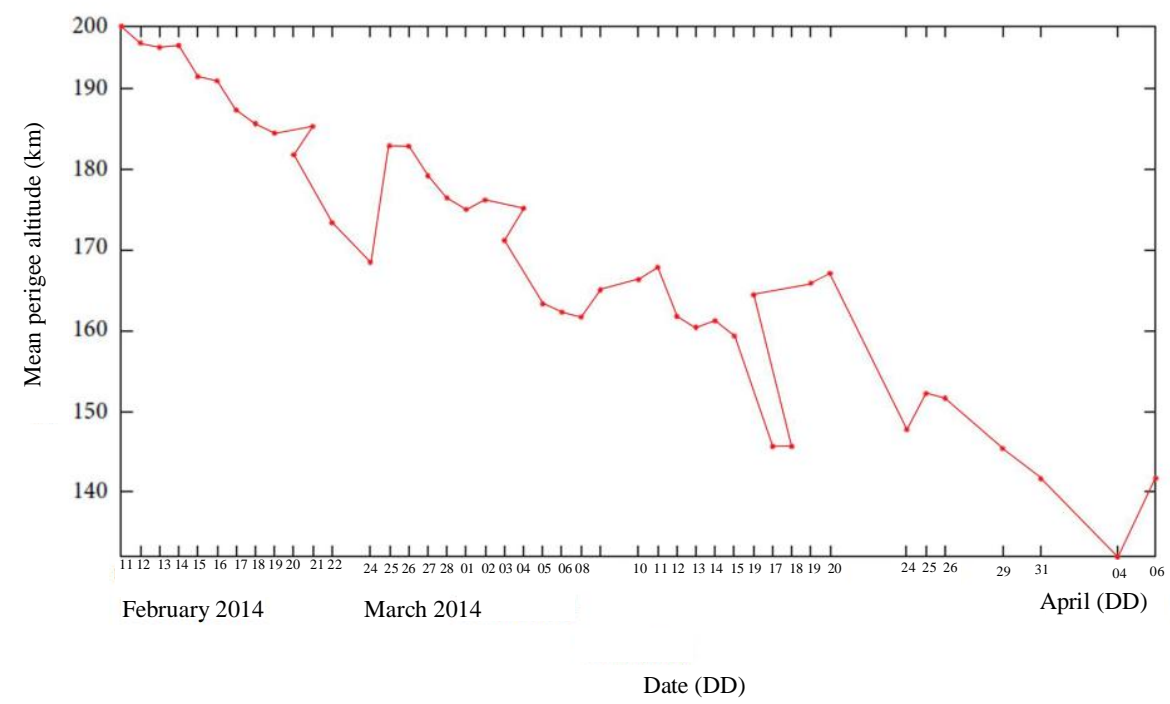

Fig. 6: Variation in computed mean perigee altitude of GSLV Rocket Body

The computation of atmospheric drag perturbations is done using the MSIS90 atmospheric density model, which includes the observed and predicted values of solar flux and geometric index. As seen in Fig. 6, the mean perigee altitude has gone below $140 \mathrm{~km}$ due to the solar perturbations which have dominated the forces acting on the object. The orbital lifetime of the upper stage of GSLV-D5 was studied, and it was found that the reentry was expected to take place between 149 to 154 days whereas the actual decay took place on June 8, 2014 (Jeyakodi and Sharma, 2014).

\section{Conclusion}

Implementation of space debris mitigation measures are essential for all space-faring nations as well as agencies to achieve the goal of clean space environment for future space exploration. India, as well as other space fairing agencies and nations, have to work together to overcome the challenges and issues related to space debris in all its future space missions. The paper provides an overview of past activities followed in design, development, and implementation of space debris mitigation measures in ISRO. The problem areas arise in the implementation of passivation of PSLV are discussed in detail. The reorbiting and decommissioning operation of INSAT-3E is explained briefly. Also, the technique utilized to minimize the orbital lifetime of GSLV-D5 in GTO has been discussed. The set of space debris mitigation guidelines developed by IADC and UNCOPUOS are strictly followed to the greater extent possible in the design and operational phases of the ISRO's spacecraft missions and launch vehicles. The proposed models, as well as techniques, were upgraded periodically to achieve the best results in the present and future missions as well. Also, the new models and tools are being designed and developed according to the advancement in technology.

\section{Acknowledgement}

The author would like to thank unknown reviewers for their thoughtful suggestions and comments.

\section{Ethics}

The author declare that there is no conflict of interest regarding the publication of this article.

\section{References}

Adimurthy, V. and A.S. Ganeshan, 2006. Space debris mitigation measures in India. Acta Astronautica, 58: 168-174.

Ananthasayanam, M.R., A. K. AnilKumar and P.V. Subba Rao, 2002. A New Stochastic Impressionistic Low Earth (SIMPLE) Model of the Space Debris Scenario, COSPAR 02-A-01772.

Ananthasayanam, M.R., A. K. AnilKumar and P.V. Subba Rao, 2003. Characterization of eccentricity and ballistic coefficient of space debris in altitude and perigee bins. IAA.5.P.04.

Ananthasayanam, M.R., A.K. Anilkumar and P.V. Subba Rao, 2006a. A new stochastic impressionistic low Earth model of the space debris scenario. Acta Astronautica, 59: 547-559. 
Ananthasayanam, M.R., A.K. Anilkumar and P.V. Subba Rao, 2006b. New Approach for the Evolution and Expansion of Space Debris Scenario. J. Spacecraft Rockets, 43: 1271-1282.

Anil Kumar, A.K. and P.V. Subba Rao, 2002. Reentry Prediction Accuracy Improvement Using Genetic Algorithm, COSPAR 02-A-01372.

Anil Kumar, A.K., M.R. Ananthasayanam and P.V. Subba Rao, 2002. A New Modeling Approach for Orbital Breakup in Space. COSPAR 02-A-01843.

Anil Kumar, A.K., M.R. Ananthasayanam and P.V. Subba Rao, 2003. On-Orbit collision probability analysis in LEO using SIMPLE model and poisson probability distribution, IAA.5.2.09.

Anilkumar, A.K., M.R. Ananthasayanam and P.V. Subba Rao, 2005. A posterior semi-stochastic low Earth debris on-orbit breakup simulation model. Acta Astronautica, 57: 733-746.

Anilkumar, A.K., Ananthasayanam, M.R., P.V. Subba Rao and V. Adimurthy, 2005. Statistical analysis of the orbital characteristics of the geo debris environment. Proceedings of the 4th European Conference on Space Debris (ESA SP-587), Darmstadt, Germany.

Anilkumar, A.K., M.R. Ananthasayanam and P.V. Subba Rao, 2007. Constant Gain Kalman Filter Approach for the Prediction of Re-Entry of Risk Objects. Acta Astronautica, 61: 831-839.

Anilkumar, A.K. and D.S. Reddy, 2009. Statistical Conjunction analysis and modeling of low-earthorbit catalogued objects. J. Spacecraft Rockets, 46: 160-167.

Anilkumar, A.K., M. Xavier Raj, M. Mutyalarao, D. Pooja and P.B. Bhanumathy et al., 2017. Performance of prediction models on reentry time prediction of CZ-2C rocket body. Proceedings of the 1st International Conference on Recent Advances in Aerospace Engineering (ICRAAE), Coimbatore.

Bandyopadhyay, P., R.K. Sharma and V. Adimurthy, 2004. Space debris proximity analysis in powered and orbital phases during satellite launch. Advances Space Res., 34: 1125-1129.

ESOC, 2018. Classification of Geosynchronous Objects, European Space Operations Center.

Ganeshan, A.S., S.C. Rathnakara, N.S. Gopinath and P. Padmanabhan, 1988. Collision Probability of Spacecraft with Man-made Debris, IAA, 88: 143-154.

Ganeshan, A.S. and M.R. Ananthasayanam, 1996. Modeling the low earth space debris distribution with limited data. IAA-96-6.3.05.
Ganeshan, A.S. and M.R. Ananathasayanam, 1997. Simulation and modeling of orbital debris environment by equivalent breakups. Advances Space Res., 19: 309-312.

Ganeshan, A.S., S. Nirmala, S.C. Rathnakara and M.R. Ananthasayanam, 2001. Ballistic parameter estimation for the equivalent break-up model. IAA01-IAA.6.5.02.

Ganeshan, A.S., R. Sharma, and A.K. Anilkumar, 2007. Recent space debris modeling activities of ISRO and salient results. Proceedings of the 58th International Astronautical Congress (IAC' 07). pp: 1786-1799.

IADC, 2007. Space Debris Mitigation Guidelines. https://www.iadconline.org/index.cgi?item=docs_pub

ISO, 2019. Space Systems - Space Debris Mitigation Requirements. International Organization for Standardization, ISO/TC 20/SC 14.

Jeyakodi David, J.F. and R.K. Sharma, 2014. Lifetime estimation of the upper stage of GSAT-14 in geostationary transfer orbit. International Scholarly Research Notices.

Kannan, M.R., P. Manikantan, D. Kumar, S. Vasanth and S. Selvamani et al., 2015. Collision avoidance assessment and mitigation measures for controlled geo/gso spacecrafts with orbital debris. Proceedings of the 7th European Conference on Space Debris ESA/ESOC.

NASA, 2019. A publication of the NASA Orbital Debris Program Office. Orbital Debris Quarterly News.

Reddy Sudheer, D., N. Gopal Reddy and A.K. Anilkumar, 2011. Modeling spatial density in low Earth orbits using wavelets and random search. Advances Space Res., 48: 1432-1440.

Sharma, R., P. Bandyopadhyay and V. Adimurthy, 2004. Consideration of lifetime limitation for spent stages in GTO. Advances Space Res., 34: 1227.1232 .

Sharma, R.K. and A.K. Anilkumar, 2005. Kalman filter approach for re-entry predictions of risk objects with k-s element equations. Proceedings of the International Astronautical Congress Paper IAC-05B6.3.05.

Shone, P.M., 2003. Design and analysis of space debris shield for spacecrafts. Thesis, M. Tech, Cochin University of Science of Technology.

Shraddha, G. and A.K. Anilkumar, 2015. Integrated model for prediction of reentry time of risk objects. J. Spacecraft Rockets, 52: 295-299. 
Santosh Kosambe / Journal of Aircraft and Spacecraft Technology 2019, Volume 3: 197.210 DOI: 10.3844/jastsp.2019.197.210

UNOOSA, 2010. Space debris mitigation guidelines of the committee on the peaceful uses of outer space. United Nations Office for Outer Space Affairs.

\section{Figure Sources}

Fig.

1:

https://orbitaldebris.jsc.nasa.gov/quarterlynews/pdfs/odqnv23i1.pdf

Fig.

2:

http://www.unoosa.org/pdf/pres/stsc2010/tech-33.pdf

Source:
Fig.

3:

Source:

http://www.unoosa.org/pdf/pres/stsc2010/tech-33.pdf

Fig.

4:

Source: http://www.jsforum.or.jp/stableuse/2016/pdf/13.\%20Dheka ne.pdf

Fig.

5:

Source:

Source:

$$
\text { http }
$$

gov/ping

6:

Source:

https://www.ncbi.nlm.nih.gov/pmc/articles/PMC4897220/ 